Document downloaded from:

http://hdl.handle.net/10251/143141

This paper must be cited as:

Polo, L.; Díaz De Greñu-Puertas, B.; Della Bella, E.; Pagani, S.; Torricelli, P.; Vivancos, J.; Ruiz Rico, M.... (11-2). Antimicrobial activity of commercial calcium phosphate based materials functionalized with vanillin. Acta Biomaterialia. 81:293-303. https://doi.org/10.1016/j.actbio.2018.09.033

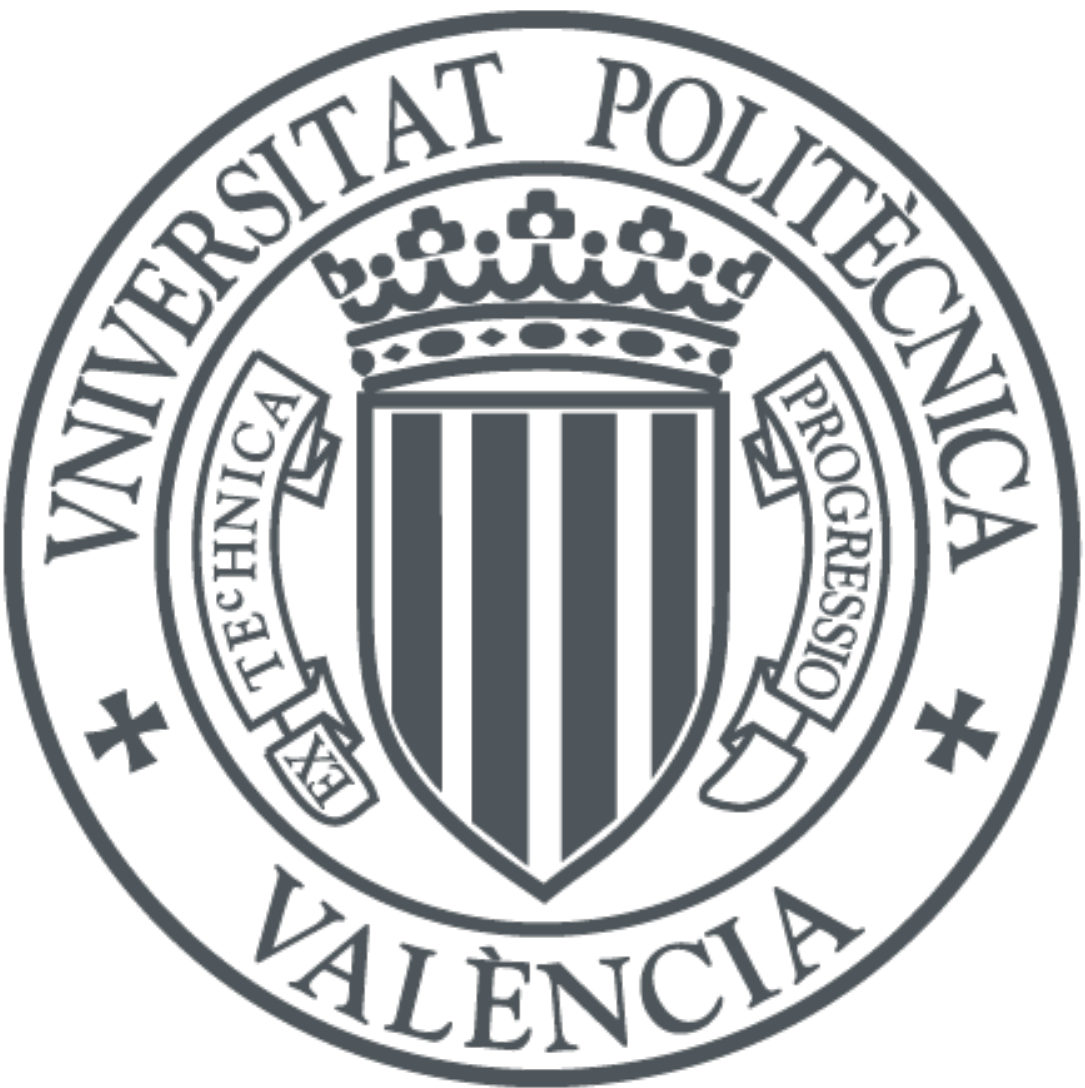

The final publication is available at

https://doi.org/10.1016/j.actbio.2018.09.033

Copyright Elsevier

Additional Information 


\section{ANTIMICROBIAL ACTIVITY OF COMMERCIAL CALCIUM PHOSPHATE BASED MATERIALS FUNCTIONALIZED WITH VANILLIN}

Lorena Polo, ${ }^{1,2}$ Borja Díaz de Greñu, ${ }^{1,2}$ Elena Della Bella, ${ }^{3}$ Stefania Pagani, ${ }^{3}$, Paola Torricelli, ${ }^{3}$ José Luis Vivancos, ${ }^{4,2}$ María Ruiz-Rico, ${ }^{5}$ José M. Barat, ${ }^{5}$ Elena Aznar, ${ }^{2,1}$ Ramón Martínez-Máñez, ${ }^{1,2} *$ Milena Fini $^{3, *}$ and Félix Sancenón ${ }^{1,2}$

${ }^{1}$ Instituto Interuniversitario de Investigación de Reconocimiento Molecular y Desarrollo Tecnológico (IDM), Universitat Politècnica de València,Universitat de València, Camí de Vera s/N, 46022, Valencia, Spain.E-mail: rmaez@qim.upv.es ${ }^{2}$ CIBER de Bioingeniería Biomateriales y Nanomedicina (CIBER-BBN), Spain. ${ }^{3}$ Laboratory of Preclinical and Surgical Studies, IRCCS Rizzoli Orthopaedic Institute, via di Barbiano 1/10, 40136 Bologna, Italy.

${ }^{4}$ GIDDP, Departamento de Proyectos de Ingeniería, Universitat Politècnica de València, Camí de Vera s/N, 46022, Valencia, Spain.

${ }^{5}$ Departamento de Tecnología de Alimentos, Universitat Politècnica de València, Camí de Vera s/N, 46022, Valencia, Spain

Keywords: biomaterials, antimicrobial, essential oils, vanillin
Abstract
Infections represent one of the most frequent causes of arthroplasty revision. Thus, design of new antimicrobial scaffolds to reduce implant rejections, bone infections and associated medical costs is highly desired. In recent years, essential oil components (EOCs) have merged as compounds with significant antimicrobial activity that can be attached to specific surfaces to enhance and prolong their antimicrobial effect. Herein calcium phosphate $\mathrm{CaP}$ regenerative materials have been coated with a vanillin derivative to combine its original bone regeneration properties with antimicrobial action of EOCs. Materials in form of microparticles and blocks were prepared and fully characterized. Clonogenic viability tests demonstrated that low concentrations of material $\left(10 \mathrm{mg} \cdot \mathrm{mL}^{-1}\right)$ resulted effective to kill $100 \%$ of E.coli DH5 $\alpha$ bacteria. 
Additionally, vanillin containing scaffolds did not display any toxic effect over cells, yet they preserve the ability to express alkaline phosphatase $(A L P L)$, collagen type 1 , chain $\alpha 1$ (COL1A1) and bone gamma-carboxyglutamic acid-containing protein or osteocalcin (BGLAP), which are genes typically expressed by osteoblasts. These results demonstrate that commercially available scaffolds can be functionalized with EOCs, achieving antimicrobial activity and open up a new approach for the treatment and prevention of infection.

\section{Statement of significance}

During the last years, the interest in bone regenerative materials with antibiotic properties has increased, since prosthesis infection is one of the most usual complications in implant surgery. In this work, we report a hybrid system composed by a calcium phosphate material (powders and scaffolds) functionalized with the derivative of an essential oil component (EOC). Our purpose was to provide the calcium phosphate material with antimicrobial activity without harming its bone regenerative capability. The obtained results were encouraging, which opens up the possibility of developing new modified materials for the prevention and treatment of bone infection.

\section{Introduction}

Over the last years the use of regenerative medicine to recover bulk and functionality of damaged tissues in trauma, large resections following primitive tumours or metastases, congenital defects, malformations or infections has increased. [1] In particular, bioceramics have been extensively studied and a large number of ceramic-based devices have been commercialised due to their suitability as bone substitutes or prosthesis 
coatings. [2] Among these ceramic-based devices, calcium phosphates $(\mathrm{CaP})$ have become essential in fields such as orthopaedics and dentistry and have been used in developing different types of prosthesis surfaces and bone cements for bone defect filling. The main reason for their suitability as bone grafts is their huge similarity to bone matrix, which is formed by a $60 \%$ of calcium phosphate. [3,4] Several different $\mathrm{CaP}$ compositions can be found in the surgery market nowadays; however, this work is centred in the use of composites of $\beta$-tricalcium phosphate $\left(\beta-\mathrm{Ca}_{3}\left(\mathrm{PO}_{4}\right)_{2}\right)$ and hydroxyapatite $\left(\mathrm{Ca}_{10}\left(\mathrm{PO}_{4}\right)_{6}(\mathrm{OH})_{2}\right)$. These materials have been proved to show good biocompatibility, bioactivity, and biodegradability. [5-7] Biocompatibility is a fundamental characteristic in surgical materials, since it is the ability to be implanted without eliciting a negative response from the host body. Lack of toxicity of calcium phosphates has been extensively tested. [8] Moreover, these materials have proved to be highly bioactive, which means that are able to participate in specific biological reactions which help the bone to regenerate. [9] CaP supports are also able to release ionic products, when implanted into the body, that can react with the phosphates present in a physiological environment allowing the formation of an apatite-like phase on the surface of the implant. $[10,11]$

The real potential of $\mathrm{CaP}$ materials arises from their osteoconductive, osteointegrative and osteoinductive capabilities. [12] Osteoconduction is the possibility of hosting bone cells and make them grow on the surface of the implant. Cell adhesion is favoured on the surface of $\mathrm{CaP}$ due to their textural properties and their ability to adsorb extracellular matrix (ECM) proteins. [13,14] Moreover, these materials are also able to integrate into the living bone (osteointegration) and recruit progenitor cells and stimulate them to develop into the bone-forming cell lineage (osteoinduction). [15-17] 
Biodegradability of surgical devices is also important, since the progressive solution of these materials avoids the necessity of performing a second intervention to remove the graft. [18] However, as well as all types of implant, even CaP shows some drawbacks. The surgery needed for the collocation of bone graft or prosthesis can lead to site morbidity and excessive inflammation, but often the major problem of these type of surgical interventions is the apparition of bone infections caused by pathogenic microorganisms found on the surface of the implants. [19] Actually, septic failure is the second most frequent cause of prosthetic failure (18.4\%). [20] Bacteria replicate and may create a biofilm on the material's surface, which makes them resistant to diverse antibiotics and hinders healing during the post-operative process. [21] For this reason, during the last years, the development of new bioceramics with antimicrobial properties against the development of bone infection processes has increased in interest. [22,23]

In the context of antibacterial substances, numerous antibiotic drugs have been developed and tested. Though oral administration may be a good solution for antibacterial treatment, high doses of drugs are required, and this can lead to antibiotic resistance or renal and liver complications. [24] For this reason, both drug delivery devices and antibacterial surfaces have been largely developed, with the purpose of achieving a local action of the drugs. [25,26] Apart from antibiotics, other substances have been studied as antimicrobials in order to avoid typical secondary effects of some drugs. [27,28] In this context, essential oil components (EOCs) have been found to present antimicrobial activity. [29] These products are naturally synthesized by plants, are fragrant and volatile, and have demonstrated to possess antimicrobial, antifungal, antiviral, insecticidal and antioxidant activity. [30-32] Particularly, it has been studied that their antimicrobial activity is due to the presence of hydroxyl moieties, which can 
interact with cellular membrane and increases its permeability, causing $\mathrm{pH}$ destabilization and membrane disruption. [33,34] Thanks to these properties, EOCs (i.e. carvacrol, eugenol, thymol, vanillin or cinnamaldehyde) have been widely used in food and pharmacologic industry. However, their high volatility and strong odor make them difficult to be applied in therapeutic fields. [35] For this reason, several studies have been carried out in order to attach essential oils to specific surfaces and to enhance and/or prolong their antimicrobial effect. [36,37]

Taking into account the above-mentioned facts, a commercial bone-regenerative material was coated in this work with an essential-oil derivative in order to combine the original bone regeneration of the material with the antimicrobial action of the EOC. For this purpose, commercially available (Surgibone@) $\mathrm{CaP}$ microparticles (powder, $\mathbf{P w}$ ) and blocks (scaffolds, $\mathbf{S c}$ ) were used as supports. Then, vanillin (1 in Scheme 1) was used as antimicrobial and grafted onto the surface of the scaffolds. In order to attach vanillin to the surface of the solids, the natural product was reacted with $1,5-$ pentanediamine (2) and sodium borohydride to yield the derivative 4-\{[(3aminopentyl)amino]methyl \}-2-methoxyphenol (3) (see Scheme 1). [38,39] Compound 3 was grafted onto the external surface of $\mathrm{CaP} \mathbf{P w}$ and $\mathbf{S c}$ using N-(3Dimethylaminopropyl)- $\mathrm{N}^{\prime}$-ethylcarbodiimide hydrochloride (EDC) yielding the final materials Pw-3 and Sc-3. Besides, the Sc support was also functionalized, using EDC, with 1,5-pentanodiamine (compound 2) for comparative purposes (Sc-2 material). A summary of the prepared solids is shown in Table 1. All functionalized materials were characterized by field emission scanning electronic microscopy (FESEM) and thermogravimetric analysis (TGA). Also, the antimicrobial behavior of the prepared materials was assessed using clonogenic viability tests against E.coli DH5 $\alpha$ bacteria. 
Finally, biocompatibility assays were carried out for checking that the original bioactive properties of the material were not damaged upon the functionalization process.

\section{Materials and methods}

\subsection{Chemicals}

Commercial Surgibone $(C$ material was purchased in the form of microspheres $(\mathbf{P w})$ and tridimensional blocks (Sc) composed by $75 \%$ hydroxyapatite and $25 \% \beta$-tricalcium phosphate. CaP powder microspheres presented a diameter of $c a .100 \mu \mathrm{m}$, whereas $\mathrm{CaP}$ scaffolds sized $10 \times 10 \times 5 \mathrm{~mm}$ were thoroughly cut in order to obtain pieces of $5 \times 5 \times$ $5 \mathrm{~mm}$.

The chemicals vanillin, 1,5-pentanediamine, imidazole, $N$-(3-dimethylaminopropyl)- $N$ 'ethylcarbodiimide hydrochloride (EDC), sodium borohydride $\left(\mathrm{NaBH}_{4}\right)$ and glutaraldehyde were purchased from Sigma-Aldrich Química S.L.

Ethylendiaminetetraacetic acid (EDTA) was purchased from Scharlab S.L (Barcelona, Spain). $\varepsilon$-Poly-L-lysine was purchased from Chengdu Jinkai Biology Engineering Co (Chengdu, China). Luria-Bertani agar medium used in bacterial experiments was provided from Laboratorios Conda (Madrid, Spain). For cell cultures, Dulbecco's minimum essential medium (DMEM), $\beta$-glicerophosphate, ascorbic acid, Neutral Red dye and Erythrosyn B were purchased from Sigma-Aldrich, UK. Fetal bovine serum (FBS) was obtained from Euroclone, (Pero, Milan, Italy). Penicillin and streptomycin were obtained from Gibco, (Life Technologies, USA). Alamar blue dye was purchased from Serotec, (Oxford, UK). Lactate dehydrogenase (LDH) cytotoxicity detection kit was obtained from Roche Diagnostics GmbH, (Manheim, Germany). LIVE/DEAD® assay kit was purchased from Molecular Probes, (Eugene, OR, USA). RNeasy Mini Kit, SYBR green PCR kit and primers set were obtained from Qiagen Srl, (Milan, Italy), and 
Superscript Vilo cDNA synthesis kit was obtained from Life Technologies, (Carlsbad, CA, USA).

\subsection{General Techniques}

FESEM images were obtained with a ZEISS ULTRA 55. Thermogravimetric analyses were carried out on a TGA/SDTA 851e Mettler Toledo balance, using an oxidant atmosphere (air, $80 \mathrm{~mL} \cdot \mathrm{min}^{-1}$ ) with a heating program consisting on a heating ramp of $10{ }^{\circ} \mathrm{C}$ per minute from 393 to $1273 \mathrm{~K}$ and an isothermal heating step at this temperature during 30 minutes.

${ }^{1} \mathrm{H}$ and ${ }^{13} \mathrm{C}-\mathrm{NMR}$ spectra were acquired in a BRUKER ADVANCE III (400 MHZ). The NMR samples were dissolved in deuterated solvents purchased from AcrosOrganics, and the residual signal of the non-deuterated solvent was used as reference. Electron impact ionization mass spectrometry (MS-EI) was performed on a Thermo Finnigan MAT SSQ710 single stage quadrupole instrument and high-resolution mass spectra (HRMS) were carried out in a TRIPLETOF T5600 (ABSciex, USA) spectrometer. Matrix-assisted laser-desorption/ionization mass spectrometry was performed on a Bruker Autoflex III Smartbeam mass spectrometer, utilizing a 2,5-dihydroxybenzoic acid (DHB) matrix.

For cell experiments, Alamar Blue fluorescence was read at $590 \mathrm{~nm}$ (excitation at 530 nm) using a Micro Plate reader (VICTOR X2030, Perkin Elmer, Milan, Italy). LDH test was measured by an iMark microplate reader, BIORAD, Milan, Italy at $490 \mathrm{~nm}$ against $655 \mathrm{~nm}$ as reference. Cell images after Neutral Red staining were taken with a standard light microscope (Nikon Eclipse, Ti-U, Nikon Italia Srl, Italy) equipped with a digital camera, at 4x and 10x magnification. Cell images after LIVE/DEAD fluorescent labelling were taken using the same microscope equipped with an epifluorescence set- 
up (Eclipse TiU, NIKON Europe BV, NITAL SpA, Milan, Italy): excitation/emission setting of $488 / 530 \mathrm{~nm}$ to detect green fluorescence (live cells) and 530/580 $\mathrm{nm}$ to detect red fluorescence (dead cells). Gene expression was evaluated by qPCR in a Light Cycler 2.0 Instrument (Roche Diagnostics, GmbH, Manheim, Germany), following a protocol which included 3 main steps: denaturation, amplification and melting curve analysis.

\subsection{Synthesis}

\subsubsection{Synthesis of 3}

A two-step synthetic procedure was used to prepare the vanillin-derivative (3). In a first step, vanillin $(\mathbf{1}, 0.76 \mathrm{~g}, 5 \mathrm{mmol})$ was dissolved in dichloromethane $(15 \mathrm{~mL})$ and added dropwise over a solution of 1,5-pentanediamine $(2,0.616 \mathrm{~mL}, 5 \mathrm{mmol})$ in dichloromethane $(25 \mathrm{~mL}$ ) under vigorous stirring (see Scheme 1). After 30 minutes of reaction, diethyl ether $(40 \mathrm{~mL})$ was added over the suspension and the pale yellow solid was filtered-off. In a second step, a portion of the resulting solid $(0.7 \mathrm{~g}, 2.97 \mathrm{mmol})$ was dissolved in ethanol $(30 \mathrm{~mL})$ and $\mathrm{NaBH}_{4}(123 \mathrm{mg}, 3.26 \mathrm{mmol})$ was slowly added in small portions. The solution was stirred for 1 hour and then ethanol was evaporated. The product was washed with ethyl acetate $(3 \times 5 \mathrm{~mL})$. Finally, acetone was added to the crude and the suspension was filtered to remove the boron salts. Evaporation of the solvent under vacuum afforded the final product $(3,522.83 \mathrm{mg}, 2.21 \mathrm{mmol}, 74 \%$ yield $)$ as a yellow solid.

${ }^{1} \mathrm{H}-\mathrm{NMR}(\mathrm{MeOD}): \delta=6.8(1 \mathrm{H}, \mathrm{s}) ; 6.62(2 \mathrm{H}, \mathrm{s}) ; 3.72(3 \mathrm{H}, \mathrm{s}) ; 3.52(2 \mathrm{H}, \mathrm{m}) ; 2.63-2.35$ $(4 \mathrm{H}, \mathrm{m}) ; 1.46-1,30(4 \mathrm{H}, \mathrm{m}), 1.28-1.18(2 \mathrm{H}, \mathrm{m})$.

${ }^{13} \mathrm{C}-\mathrm{NMR}$ (MeOD): $\delta=149.11 ; 147.14,131.59 ; 122.41 ; 116.10 ; 113.29 ; 65.33 ; 56.33$, $54.27 ; 42.16 ; 32.89 ; 30.05 ; 25.66$.

HRMS, calculated for $\mathrm{C}_{13} \mathrm{H}_{22} \mathrm{~N}_{2} \mathrm{O}_{2} 238.1681$, found $239.1754\left(\mathrm{M}+\mathrm{H}^{+}\right)$. 


\subsubsection{Preparation of Pw-3, Sc-3 and Sc-2}

For the functionalization of the CaP materials (Pw and Sc), vanillin derivative 3 (523 $\mathrm{mg}, 2.21 \mathrm{mmol})$ or 1,5-pentanediamine $(2,257 \mathrm{mg}, 2.51 \mathrm{mmol})$ was dissolved in a solution of imidazole in water $(10 \mathrm{~mL}, 0.1 \mathrm{M})$. On the other hand, phosphate groups of Pw (300 mg) or Sc (6 pieces) were activated by immersion of the materials into a solution of EDC (1.25 g, $6.52 \mathrm{mmol})$ in PBS-EDTA 0.01 M buffer (10 mL). Finally, the solution of $\mathbf{2}$ or $\mathbf{3}$ was added to the scaffolds, and the samples were stirred for 24 hours at $37^{\circ} \mathrm{C}$. Then, Pw-3, Sc-3 and Sc-2 were washed with water and acetone and dried under vacuum.

\subsection{Bacterial viability assay}

\subsubsection{E. coli DH5 $\alpha$ culture conditions}

For bacterial viability studies, Escherichia coli (E. coli) DH5 $\alpha$ cell culture was used. Bacteria cells were maintained in glycerol $15 \%$ at $-80{ }^{\circ} \mathrm{C}$. For the assays, cells were grown for 24 hours at $37^{\circ} \mathrm{C}$ and under constant stirring with $5 \mathrm{~mL}$ of Luria-Bertani Broth medium. Bacteria from $1 \mathrm{~mL}$ culture were collected by centrifugation for 30 seconds at $13000 \mathrm{rpm}$ and re-suspended in $1 \mathrm{~mL}$ of PBS at $\mathrm{pH}$ 7.6.

\subsubsection{Bacterial viability assay with $P w-3$}

E.coli bacteria were treated with different amounts of $\mathbf{P w}-\mathbf{3}$ in order to determine its antimicrobial potential. For this purpose, a suspension of $10^{3}$ cells $\cdot \mathrm{mL}^{-1}$ was stirred in the presence of 50, 5, 2, 1, 0.5, 0.35, 0.1, 0.05, 0.025, 0.005 and $0 \mathrm{mg} \cdot \mathrm{mL}^{-1}$ of $\mathbf{P w} \mathbf{- 3}$ for 15 minutes at $37^{\circ} \mathrm{C} .100 \mu \mathrm{L}$ of the suspensions were seeded in LB plates (3\% agar) and incubated at $37{ }^{\circ} \mathrm{C}$ for 24 hours. Then, colony formation units (CFU) were counted. In order to compare the antibacterial activity of the materials with that of free vanillin (1), 
diamine (2) and the synthesized molecule (3), the same experiment was carried out with solutions of equivalent concentrations of $\mathbf{1}, \mathbf{2}$, and $\mathbf{3}$. A control experiment was also carried out with $\mathbf{P w}$ (without any functionalization) in order to demonstrate that the toxic effect was not due to the raw material.

\subsubsection{Bacterial viability assay with Sc, Sc-2, Sc-3, and 1, 2, and 3}

For testing the antimicrobial potential of the functionalized CaP scaffolds, suspensions of $10^{3}, 10^{4}$ and $10^{5}$ cells $\cdot \mathrm{mL}^{-1}$ were prepared. Then, $1 \mathrm{~mL}$ of each suspension was stirred in the presence of $\mathbf{S c - 3}$ for 15 minutes at $37^{\circ} \mathrm{C}$. The suspensions were diluted with PBS in order to obtain a cell concentration easy to quantify, and $100 \mu \mathrm{L}$ of the new dilutions were seeded in LB plates ( $3 \%$ agar) and incubated at $37^{\circ} \mathrm{C}$ for 24 hours. Then, colony formation units (CFU) were quantified. In order to determine that the antimicrobial behavior was due to the presence of the modified vanillin molecule, similar experiments were carried out in the presence of non-functionalized scaffolds $(\mathbf{S c})$, diaminefunctionalized scaffolds (Sc-2), and in the presence of equitoxic amounts of solutions of 1, 2, and 3. Triplicate experiments were carried out for each sample. Finally, untreated suspensions of $10^{3}, 10^{4}$ and $10^{5}$ cells $\cdot \mathrm{mL}^{-1}$ were also used as a control.

\subsubsection{Preparation of bacteria for FESEM}

Bacteria treated with Sc and with Sc-3 were filtered with PTFE $0.22 \mu \mathrm{m}$ filters previously treated with a solution of $0.1 \% \varepsilon$-poly-L-lysine. Then, the filters were submerged into a solution of $2.5 \%$ glutaraldehyde for 1 hour in order to fix the bacteria. Dehydration was carried out by submerging the filters in graded ethanol solution series $(50,70,80,95$ and $100 \%)$ for 10 minutes each. The samples were dried by critical point drying technique and coated with carbon for FESEM examination.

\subsection{Cytotoxicity assays}




\subsubsection{Cell culture conditions}

L929 fibroblast-like cells from American Type Culture Collection (ATCC-LGC

Standards S.r.L., Milan, Italy) were maintained and expanded in DMEM supplemented with $10 \% \mathrm{FBS}, 100 \mathrm{U} \cdot \mathrm{ml}^{-1}$ penicillin and $100 \mu \mathrm{g} \cdot \mathrm{ml}^{-1}$ streptomycin in standard conditions $\left(37^{\circ} \mathrm{C}, 5 \% \mathrm{CO}_{2} / 95 \%\right.$ air, humidified atmosphere).

\subsubsection{In vitro cell toxicity test}

Before conducting cell toxicity experiments, samples (Sc-3, Sc-2, and $\mathbf{S c}$ as reference) were washed with phosphate buffer solution (PBS), and sterilized for 15 minutes in ethanol $85 \%$ and then $15 \mathrm{~min}$ in ethanol $70 \%$, by stirring. To assess cytotoxicity, L929 fibroblast-like cells were seeded at the density of $1.5 \cdot 10^{4}$ cells $\cdot \mathrm{cm}^{-2}$ in 24 -well plate and incubated for 24 hours. Triplicate wells were seeded for each sample, including negative and positive controls. The day after $\mathbf{S c}, \mathbf{S c - 2}$ and $\mathbf{S c - 3}$ materials were carefully placed on the cell layer, ensuring that the specimens covered at least one tenth of the well bottom surface. A positive control (CTR+) was set up by adding $0.05 \%$ phenol solution to the growth medium, and the negative control (CTR-) was represented by the only cell culture in complete DMEM.

In order to quantify cell viability at 24 and 48 hours, Alamar blue dye was added (1:10 v/v) to each culture, after removing the scaffold from wells, and incubated for 4 hours at $37^{\circ} \mathrm{C}$. The reagent is a dye that incorporates a redox indicator which changes its color in response to the chemical reduction of the growth medium resulting from cell growth. The results, in term of fluorescence, were expressed as relative fluorescence units (RFU).

At the same time points, the cell cultures supernatants were collected to detect LDH, which is a typical enzyme released by suffering and death cells. This procedure was performed following manufacturer's instruction. The developed colour, due to the 
activity of LDH, was spectrophotometrically read and the percentage of cytotoxicity for each sample was calculated with the following formula:

$$
\frac{\square \square \square_{\text {sample }}-\mathrm{ABS}_{\square \square \square-}}{\mathrm{ABS}_{\mathrm{CTR}+}-\mathrm{ABS}_{\mathrm{CTR}-}} \square 100
$$

To observe the morphology of viable cells, a solution of $0.33 \%$ Neutral Red dye in DMEM was added to each culture well $(1: 10 \mathrm{v} / \mathrm{v})$ and incubated for 20 minutes at 37 ${ }^{\circ} \mathrm{C}$. Viable cells typically incorporate the Neutral Red dye and preserve a normal morphology, while dead cells do not perform Neutral Red intake. Ten images of each culture were captured using a standard light microscope. The more significant images were analyzed according to the score reported below (UNI EN ISO 10993-5).

\subsection{Bioactivity assays}

\subsubsection{Cell culture conditions}

Before conducting the biocompatibility assay, all samples (Sc-3, Sc-2, and $\mathbf{S c}$ as reference) were sterilized in ethanol as previously described. MG-63 human osteoblastlike cells from American Type Culture Collection (ATCC-LGC Standards S.r.L., Milan, Italy) were used for biocompatibility experiments. Cells were maintained and expanded in DMEM supplemented with $10 \% \mathrm{FBS}, 100 \mathrm{U} \cdot \mathrm{ml}^{-1}$ penicillin and $100 \mu \mathrm{g} \cdot \mathrm{ml}^{-1}$ streptomycin, $\beta$-glicerophosphate $\left(10^{-4} \mathrm{M}\right)$ and ascorbic acid $\left(50 \mu \mathrm{g} \cdot \mathrm{ml}^{-1}\right)$ in standard conditions $\left(37^{\circ} \mathrm{C}, 5 \% \mathrm{CO}_{2} / 95 \%\right.$ air, humidified atmosphere). At $80-90 \%$ of confluence, the cells were detached by trypsinization and counted with Erythrosyn B vital dye to exclude dead cells. A suspension of $3 \cdot 10^{4}$ cells in $30 \mu$ of medium was dropwise seeded on the surface of the scaffolds (equivalent to $10^{5}$ cells $\cdot \mathrm{cm}^{-2}$ ). Cell dispersion on the well bottom was minimized by carefully pipetting and repeating cell deposition and recovery. After 3 hours of incubation, $1.5 \mathrm{~mL}$ of medium were 
added to each well, so fully embedding the scaffolds. The same number of cells in 1.5 $\mathrm{ml}$ of medium was also seeded on polystyrene plates as control (CTR). The engineered constructs and the controls were cultured for 21 days by refeeding the culture medium twice a week.

\subsubsection{Cell viability and morphology}

In order to quantify cell viability at 24 hours, 1, 2, and 3 weeks, Alamar blue test was performed, as previously described for cell toxicity evaluation. Cell morphology and sample colonization were observed by LIVE/DEAD® assay according to the manufacturer's instructions. This mixture of fluorescent dyes includes calcein (suitable to label the vital cells), and ethidium homodimer (EthD-1, which goes through the damaged membranes of dead cells). Samples were visualized using an inverted microscope equipped with an epifluorescence set-up. The green and red fluorescence was typical of live and dead cells respectively. Five images of each sample and control, at all time points, were captured using the digital camera, at $4 \mathrm{x}$ and $10 \mathrm{x}$ magnification.

\subsubsection{Gene expression analysis}

Gene expression was observed at 1,2 and 3 weeks of culture. Total RNA was extracted by engineered constructs and controls using the commercial RNeasy Mini Kit and reverse transcribed using the Superscript Vilo cDNA synthesis kit. cDNA was diluted to

the final concentration of $5 \mathrm{ng} \cdot \mu^{-1}$, and $10 \mathrm{ng}$ of each sample were tested in duplicate. Gene expression was evaluated by qPCR, using the SYBR green PCR kit in a Light Cycler 2.0 Instrument. The protocol included a denaturation cycle at $95^{\circ} \mathrm{C}$ for 15 minutes, 25 to 40 cycles of amplification and a melting curve analysis to check for amplicon specificity. The following primer sets were used: GAPDH (forward: 5'CTCTACCCACGGCAAGTTCAAC-3', reverse: 5'GACATACTCAGCACCAGCATCAC-3'), ALPL (QuantiTect Primer Assay 
Hs_ALPL_1_SG), COL1A1 (QuantiTect Primer Assay Hs_COL1A1_1_SG), BGLAP (QuantiTect Primer Assay Hs_BGLAP_1_SG). The annealing temperatures were $55^{\circ} \mathrm{C}$ for all the primer sets except for GAPDH at $60^{\circ} \mathrm{C}$. The mean threshold cycle was determined for each sample and used for the calculation of relative expression using the Livak method $\left(2^{-\Delta \Delta C t}\right)$, with GAPDH as reference gene and Sc samples as calibrators at each experimental time. [40]

\subsection{Statistics}

The statistical evaluation of data was performed using the software package SPSS/PC+ StatisticsTM 23.0 (SPSS Inc., Chicago, IL). The study is the results of three independent experiments and data are reported as mean standard deviations (SD) at a significance level of $\mathrm{p}<0.05$. After having verified normal distribution and homogeneity of variance, a one-way ANOVA was done for comparison between groups. Finally, post hoc multiple comparison test (Dunnett) was performed to detect significant differences among groups and controls. Student's t-test was used for the comparison between two groups.

\section{Results and discussion}

\subsection{Synthesis and characterization of the functionalized CaP materials}

In this work, two different commercially available CaP materials (as powder $\mathbf{P w}$ or as scaffold Sc) were functionalized with an EOC derived molecule with the purpose of combining bone regenerative capabilities of calcium phosphates [41] with the antimicrobial activity of the vanillin essential oil. Vanillin (1 in Scheme 1) was selected as an EOC due to its well-known antimicrobial activity. The presence of an aldehyde functional group in $\mathbf{1}$ ensures an easy grafting onto the CaP supports through the phosphate moieties. For this purpose, vanillin was derivatized using a two-step protocol 
(see Scheme 1). In a first step, vanillin was reacted with 1,5-pentanediamine (2) yielding the corresponding Schiff base. Then, in a second step, the imine bond of the Schiff base was reduced with sodium borohydride yielding vanillin derivative 3 , which contains an amine moiety that allows the grafting of this compound onto the $\mathrm{CaP}$ supports. Compound 3 was fully characterized using ${ }^{1} \mathrm{H},{ }^{13} \mathrm{C}-\mathrm{NMR}$ and HRMS (see Materials and Methods section). CaP powder microparticles (Pw) and scaffolds (Sc) were finally functionalized with vanillin derivative $\mathbf{3}$ through phosphoramidite formation using EDC as coupling agent. This procedure yielded solids Pw-3 and Sc-3. Besides, for comparative purposes, CaP scaffolds were also functionalized with 1,5pentanediamine (2) yielding solid Sc-2. Table 1 showed the five materials used in this paper.

The prepared materials were fully characterized using typical techniques [42-44] such as thermogravimetric analysis (TGA) and field emission scanning electronic microscopy (FESEM). TGA analysis of solids Pw-3, Sc-2 and Sc-3 were carried out in order to assess the organic matter anchored onto their surface (see Table 2). The obtained thermogravimetric curves presented three different steps (See S1 in Supplementary Material). The first step, from 0 to $100{ }^{\circ} \mathrm{C}$, corresponds to the loss of adsorbed water or organic solvents (a). The second step, from $100{ }^{\circ} \mathrm{C}$ to $800{ }^{\circ} \mathrm{C}$, quantifies the burning of organic matter attached to the CaP scaffolds (b). The last step, from $800{ }^{\circ} \mathrm{C}$ to $1000{ }^{\circ} \mathrm{C}$, could be ascribed to a mass loss associated with the hydroxyapatite decomposition into tetracalcium phosphate (TTCP) and $\alpha$-tricalcium phosphate $(\alpha \mathrm{TCP})$, which finished at $1350{ }^{\circ} \mathrm{C}(\mathrm{c})$. The higher degree of functionalization was observed for Pw-3 material (6.86 $\mathrm{mg} \cdot \mathrm{g}^{-1}$ of solid) whereas those obtained for Sc-3 and Sc-2 were lower and quite similar between them (3.46 and 3.14 $\mathrm{mg} \cdot \mathrm{g}^{-1}$ solid respectively). The larger organic matter content of $\mathbf{P w} \mathbf{- 3}$, when compared 
to that for $\mathbf{S c - 2}$ and $\mathbf{S c - 3}$, is most likely related with its higher specific surface due to its microparticulated nature. Also, thermogravimetric studies with raw $\mathbf{P w}$ and $\mathbf{S c}$ materials were carried out. As expected, thermogravimetric curves of $\mathbf{P w}$ and $\mathbf{S c}$ only showed the loss weight associated with hydroxyapatite decomposition.

In order to demonstrate that the surface of the materials did not undergo substantial changes during the functionalization process, FESEM studies were undertaken. FESEM images of Sc, Sc-2 and Sc-3 are shown in Figure 1. As could be seen, both solids present a uniform porous structure typical of the $\mathrm{CaP}$ materials. Besides, the surface of the materials before and after functionalization with vanillin derivative $\mathbf{3}$ was quite similar. This fact indicated that the functionalization process did not change substantially the structure of the initial raw material.

\subsection{Assessment of the antimicrobial behavior}

After the characterization of the prepared materials their antimicrobial behaviour against E.coli bacteria was tested. Solid Pw-3 was selected and used for the quantification of its dose dependent antibacterial activity. For this purpose, suspensions of bacteria $\left(10^{3}\right.$ $\mathrm{CFU} \cdot \mathrm{mL}^{-1}$ ) were stirred in the presence of different amounts of Pw-3. The suspensions were diluted and seeded and, after 24 hours of incubation, CFU were quantified. The same experiments were carried out with equitoxic concentrations vanillin derivative $\mathbf{3}$, 1,5-pentanediamine (2) and vanillin (1), to compare the antibacterial activity of each compound. Moreover, bacteria were also treated with different concentrations of the non-functionalized microparticles (Pw) as a control. The obtained results are shown in Figure 2, which shows the decrease on bacterial viability upon treatment with increasing concentrations of Pw-3, 3, 2, and 1. As could be seen in Figure 2, equitoxic concentrations of the vanillin derivative $\mathbf{3}$ attached onto the microparticles surface (solid 
Pw-3) were more effective in killing E. Coli bacteria than free vanillin (1), free 1,5pentanediamine (2) and free 3 . Table 3 showed the $\mathrm{EC}_{50}$ (half maximal effective concentration, which represents the amount of drug necessary to achieve $50 \%$ of bacterial death) values obtained for 1, 2, 3 and Pw-3. The less effective compound was vanillin $\left(1, \mathrm{EC}_{50}\right.$ of $\left.6.33 \mathrm{mg} \cdot \mathrm{mL}^{-1}\right)$ probably due to its volatility. The antibacterial activity of 2 and $\mathbf{3}$ were quite similar with $\mathrm{EC}_{50}$ values of 0.092 and $0.083 \mathrm{mg} \cdot \mathrm{mL}^{-1}$ respectively. However, the $\mathrm{EC}_{50}$ for $\mathbf{P w}-\mathbf{3}\left(0.015 \mathrm{mg} \cdot \mathrm{mL}^{-1}\right)$ was approximately one order or magnitude lower than that found for free vanillin derivative 3 . This enhanced antibacterial activity of $\mathbf{P w - 3}$ could be ascribed to a marked reduction in the volatility of derivative $\mathbf{3}$ upon its grafting onto the surface of the microparticles. Besides, in order to check that the antibacterial activity determined for $\mathbf{P w}-\mathbf{3}$ was due to the grafted vanillin derivative $\mathbf{3}$ and not to the solid itself, the same antimicrobial experiments were carried out with Pw. These experiments indicated that $\mathbf{P w}$ was not toxic for bacteria as negligible changes in bacterial viability were observed (data not shown).

Once the antibacterial effect of the Pw-3 microspheres had been tested, E.coli bacteria were treated with the functionalized scaffolds in order to verify that the tridimensional scaffolds also displayed an antimicrobial activity. For this experiment, different suspensions of $10^{3}, 10^{4}$ and $10^{5} \mathrm{CFU} \cdot \mathrm{mL}^{-1}$ were treated with $\mathbf{S c - 3}$. As control bacteria were incubated with the non-functionalized scaffold (Sc). In addition, the diaminefunctionalized scaffold (Sc-2) was also tested in order to study the effect of the diamine and compare it with the effect of vanillin. Also, equitoxic concentration of vanillin derivative $\mathbf{3}$ and free vanillin (1) were tested. Finally, untreated suspensions of bacteria $10^{3}, 10^{4}$ and $10^{5} \mathrm{CFU} \cdot \mathrm{mL}^{-1}$ were used as a control. The obtained results, in terms of bacterial viability, are shown in Figure 3. As could be seen, the raw scaffold (Sc) did 
not affect significantly bacterial viability. On the other hand, as expected, Sc-3 solid induced a marked reduction in E. coli viability (ca. $90 \%$ for $\left.10^{3} \mathrm{CFU} \cdot \mathrm{mL}^{-1}\right)$. Besides, toxicity of Sc-3 scaffold decreased at higher bacteria concentration ( $c$ a. $60 \%$ for $10^{5}$ $\mathrm{CFU} \cdot \mathrm{mL}^{-1}$ ). These results clearly indicated that antibacterial activity of $\mathbf{S c - 3}$ solid was due by the grafted vanillin derivative 3. Similar results were obtained when using Sc-2 solid (CaP scaffold functionalized with 1,5-pentanediamine). On the other hand, vanillin derivative $\mathbf{3}$ showed high toxicity for the three bacteria concentrations tested while free vanillin (1) showed a poor antibacterial activity. Remarkable, Sc-3 solid was able to induce a marked bacterial viability reduction when compared with equitoxic concentrations of free vanillin (1) demonstrating that grafting of EOCs onto the surface of CaP scaffolds increase their antibacterial properties (most probably because the immobilization process prevents volatilization).

In order to study the effect of Sc and Sc-3 on E. coli morphology, FESEM images were taken (see Figure 4). As could be seen, the morphology of E. coli bacteria treated with the raw non-functionalized scaffold (Sc) did not present any structural damage (Figure 4A). However, when bacteria were treated with Sc-3 scaffold, the external membrane was clearly damaged (Figure 4B), followed by shrinking (Figure 4C) and, finally, bacterial death (Figure 4D). The bacterial death observed with $\mathbf{S c - 3}$ supported the mechanism described in bibliography,[27] according to which the antimicrobial power of EOCs comes from their ability of disrupting cell membrane by damaging ion exchange channels. This makes the cell membrane more permeable and allows the leaking of cytoplasmatic components, leading to the shrinking of the cell wall and consequent cell death.

\subsection{Cytotoxicity assays}


After assessing the antimicrobial activity of the prepared scaffold-based materials (Sc-2 and Sc-3), cytotoxicity experiments were carried out. To perform these experiments, L929 fibroblast-like cells were cultured in the presence of Sc, Sc-2 and Sc-3 scaffolds. Besides, positive (CTR+, addition of $0.5 \%$ phenol solution to the growth medium) and negative (CTR-, cell culture in DMEM) controls were also performed. Cell viability was quantified by Alamar Blue assay, and the obtained results after 24 and 48 hours are shown in Figure 5A. As could be seen, the percentage of viability, compared with negative control (taken as $100 \%$ ), for samples treated with Sc, Sc-2 and Sc-3 was $c a$. $100 \%$, which means that no cytotoxic effect was found with the bare and functionalized scaffolds.

Levels of lactate dehydrogenase $(\mathrm{LDH})$ in the cell culture supernatant were also measured in order to assess the effect of $\mathbf{S c}, \mathbf{S c - 2}$ and $\mathbf{S c - 3}$ scaffolds in cell viability. As LDH is an intracellular enzyme, high concentrations in the culture supernatant would mean cellular membrane damage. The obtained results are shown in Figure 5B. As could be seen, negligible amounts of LDH (similar to that presented by negative control) were observed in the culture supernatants of L929 cells treated with Sc, Sc-2 and Sc-3 materials after 24 hours. Besides, after 48 hours, the amounts of LDH released were lower than $20 \%$ (materials are classified as toxic when extracellular LDH levels are higher than $30 \%$ ).

Furthermore, to confirm the cell viability quantified by Alamar Blue and to appreciate the cell morphology, also the Neutral Red staining test was performed. Images of L929 cells cultured in the presence of $\mathbf{S c}, \mathbf{S c - 2}$ and $\mathbf{S c - 3}$ were taken and compared to those obtained from negative and positive controls (Figure 6). All cultures in presence of the scaffolds (Sc, Sc-2 and Sc-3) showed a noticeable cell red staining, similar to that appreciable in the negative control (CTR-), after 24 and 48 hours of incubation. This red 
staining indicated a high L929 cell viability as only living cells are able to uptake Neutral Red. Besides, as could be seen in Figure 6, L929 cell morphology did not show any significant change supporting the previous results. On the other hand, L929 cells in positive control $(\mathrm{CTR}+)$ conditions showed no red staining and its morphology clearly indicated cell death. In conclusion, no significant cytotoxic effect on L929 cells was observed in the presence of the three scaffolds (Sc, Sc-2 and Sc-3).

\subsection{Bioactivity assays}

Once assessed the antimicrobial behaviour and the cytotoxicity of the functionalized scaffolds, bioactivity assays were performed to test if the prepared supports were suitable for enhancing bone regeneration. For this purpose, MG-63 human osteoblastlike cells were directly seeded on the surface of $\mathbf{S c}, \mathbf{S c - 2}$ and $\mathbf{S c - 3}$ and the samples were studied for 3 weeks.

In a first step, cell viabililty in $\mathbf{S c}, \mathbf{S c - 2}$ and $\mathbf{S c - 3}$ treated groups was studied using Alamar Blue dye test after 24 hours, 1, 2 and 3 weeks. The obtained results are shown in Figure 7. As could be seen, a very similar trend of the cell viability for the three scaffolds tested (i.e. Sc, Sc-2 and Sc-3) was observed with a regular increase until 2 weeks. After this time viabililty remained constant, probably due to the possible cell confluence achieved that could have slowed its growth. On the other hand, the cell viability of the control was higher than that observed with $\mathbf{S c}, \mathbf{S c - 2}$ and $\mathbf{S c - 3}$ (data not shown) probably due to the difference of the seeding areas. The seeded area corresponded to $0.5 \mathrm{~cm}^{2}$ in the case of the scaffods, while the bottom well area was 2 $\mathrm{cm}^{2}$ in the case of the controls. Neverthless, the control group represent an "internal control" for checking the cell culture conditions; the smooth and flat surface of the culture well, in contrast with the rough, irregular and partially porously structure of the CaP-based scaffolds, makes the Sc the correct control for the others materials. Statistical 
analysis showed the same difference among controls and all experimental materials at each timepoints $(* * * \mathrm{p}<0.0005)$.

In order to confirm the viability findings obtained by Alamar test, MG-63 human osteoblast-like cells morphology and sample colonization were also observed. For this purpose, three samples of $\mathbf{S c}, \mathbf{S c - 2}$ and $\mathbf{S c - 3}$ were stained using the LIVE/DEAD ${ }^{\circledR}$ kit. Figure 8 shows cell colonization of the scaffolds surface, displaying living and dead cells in green and red colour, respectively. The porous architecture of the scaffolds can be observed in the images and, after 24 hours, only a small number of cells adhered to the inner surface of the macropores. After 1 and 2 weeks of culture, cells progressively covered the surface of the scaffolds in all replicates. At this respect, no dead cells were found in the presence of $\mathbf{S c}$ or $\mathbf{S c - 3}$ after 1 and 3 weeks. However, some dead cells were observed only on Sc-2 scaffold, possibly due to slight toxicity of the amine derivatives after a long-time exposure. At the end of the experimental time, the scaffold surfaces were observed to be totally covered by cells, which indicated that scaffold colonization is possible after surface functionalization. Dead cells are also observed, probably due to the long cell exposure to a confluence environment in a static system. However, as confirmed by Alamar Blue results, the quantity of dead cells was minimum and did not affect total cell viability.

Finally, to broaden the knowledge about these new functionalized scaffolds in terms of osteoinductivity, the expression of $A L P L, C O L 1 A 1$ and $B G L A P$ genes (typically expressed by osteoblasts) was evaluated. The levels of gene expression are shown in Figure 9. As could be observed there was no significant differences in gene expression for the three scaffolds tested (Sc, Sc-2 and Sc-3). In particular, Sc-3 scaffold induced a slightly enhancement in gene expression over time compared with the corresponding controls, despite the ALPL fluctuation over time. This overall behavior allowed to state 
that the chemical modifications of the hydroxyapatite-based scaffold did not affect its ability to stimulate the bone matrix synthesis.

\section{Conclusions}

In conclusion, new vanillin-functionalized microspheres (Pw-3) or scaffolds (Sc-3) with antimicrobial features have been developed. The synthetic vanillin derivative $\mathbf{3}$ or diamine 2 were covalently anchored onto the CaP microparticles and scaffolds. No substantial changes in the materials were observed during the functionalization processes according to TGA and FESEM studies. Moreover, their antibacterial activity was tested against $E$. coli bacteria. Treatment with $10 \mathrm{mg} \cdot \mathrm{mL}^{-1}$ of solid $\mathbf{P w} \mathbf{- 3}$ resulted in $100 \%$ bacterial death, whereas scaffolds Sc-2 and Sc-3 also caused bacterial death. In addition, Sc, Sc-2 and Sc-3 scaffolds were no cytotoxic and highly biocompatible, yet they did not display any toxic effect over non-bacterial cells, because they preserve the ability to express the genes $A L P L, C O L 1 A 1$ and BGLAP. In short, these results demonstrate that commercially available scaffolds can be easily functionalized with EOCs, achieving outrageous antimicrobial activity and opening up a new approach for the treatment and prevention of infections. This study represents the first step to identify new antibacterial strategy based on the use of EOCs; even if the findings are encouraging in all the investigated aspects, other insights are mandatory before the clinical use of the materials, such as an in vivo phase to confirm their efficacy and the absence of toxicity.

\section{Acknowledgements}

The authors thank the Spanish Government for projects MAT2015-64139-C04-01-R (MINECO/FEDER). Also, Generalitat Valenciana (project PROMETEOII/2014/047) is 
acknowledged for their support. L. P. thanks Universitat Politècnica de València for her FPI and mobility grants. B.D. thanks to Ministerio de Economia, Ciencia y

Competitividad for his Juan de la Cierva-Formación fellowship. The authors also thank the Electron Microscopy Service at the UPV for their support. The authors are grateful to IRCCS Rizzoli Orthopaedic Institute (funds 5 X 1000 year 2015, cod. 6879).

\section{References}

[1] S. Oh, N. Oh, M. Appleford, J.L. Ong, Bioceramics for Tissue Engineering Applications: A Review, Am. J. Biochem. Biotechnol., 2 (2006) 49-56.

[2] L.L. Hench, I. Thompson, Twenty-first century challenges for biomaterials, J. R. Soc. Interface. 7 Suppl 4 (2010) 379-91.

[3] J. Ferguson, M. Diefenbeck, M. McNally, Ceramic Biocomposites as Biodegradable Antibiotic Carriers in the Treatment of Bone Infections, J. Bone Jt. Infect. 2 (2017) 38-51.

[4] F. Baino, G. Novajra, C. Vitale-Brovarone, Bioceramics and Scaffolds: A Winning Combination for Tissue Engineering, Front. Bioeng. Biotechnol. 3 (2015).

[5] V. Uskoković, V.M. Wu, Calcium phosphate as a key material for socially responsible tissue engineering, Materials (Basel). 9 (2016) 434.

[6] N. Eliaz, N. Metoki, Calcium phosphate bioceramics: A review of their history, structure, properties, coating technologies and biomedical applications, Materials (Basel). 10 (2017) 334.

[7] V.S. Kattimani, S. Kondaka, K.P. Lingamaneni, Hydroxyapatite: Past, Present, and Future in Bone Regeneration, Bone Tissue Regen. Insights. 7 (2016) 9-19.

[8] A.H. Choi, B. Ben-Nissan, R.C. Conway, I.J. Macha, Advances in Calcium Phosphate Nanocoatings and Nanocomposites, in: Besim Ben-Nissan (Eds.) Advances in Calcium Phosphate Biomaterials. Springer Series in Biomaterials Science and Engineering, vol 2. Springer, Berlin, Heidelberg, 2014, pp. 489-509.

[9] K. de Groot, J.G.C. Wolke, J.A. Jansen, Calcium phosphate coatings for medical implants, Proc. Inst. Mech. Eng. Part H J. Eng. Med. 212 (1998) 137-147.

[10] Q. Liu, S. Huang, J.P. Matinlinna, Z. Chen, H. Pan, Insight into biological apatite: Physiochemical properties and preparation approaches, Biomed Res. Int. 2013 (2013). 
[11] R.Z. LeGeros, Biodegradation and bioresorption of calcium phosphate ceramics, Clin. Mater. 14 (1993) 65-88.

[12] H. Yuan, Z. Yang, Y. Li, X. Zhang, J.D. De Bruijn, K. De Groot, Osteoinduction by calcium phosphate biomaterials, J. Mater. Sci. Mater. Med. 9 (1998) 723-726.

[13] J. Wilson, S.B. Low, Bioactive ceramics for periodontal treatment: comparative studies in the Patus monkey, J. Appl. Biomater. 3 (1992) 123-129.

[14] V.A. Dubok, Bioceramics - Yesterday, today, tomorrow, Powder Metall. Met. Ceram. 39 (2000) 381-394.

[15] S. Weiner, H.D. Wagner, THE MATERIAL BONE: Structure-Mechanical Function Relations, Annu. Rev. Mater. Sci. 28 (1998) 271-298.

[16] S. Samavedi, A.R. Whittington, A.S. Goldstein, Calcium phosphate ceramics in bone tissue engineering: A review of properties and their influence on cell behavior, Acta Biomater. 9 (2013) 8037-8045.

[17] D.M. Dohan Ehrenfest, P.G. Coelho, B.S. Kang, Y.T. Sul, T. Albrektsson, Classification of osseointegrated implant surfaces: Materials, chemistry and topography, Trends Biotechnol. 28 (2010) 198-206.

[18] F.C.J. van de Watering, J.J.J.P. van den Beucken, R.P.F. Lanao, J.G.C. Wolke, J.A. Jansen, Biodegradation of Calcium Phosphate Cement Composites BT, in: N. Eliaz (Ed.), Degradation of Implant Materials, Springer New York, New York, 2012: pp. 139-172.

[19] J. Esteban, J. Cordero-Ampuero, Treatment of prosthetic osteoarticular infections., Expert Opin. Pharmacother. 12 (2011) 899-912.

[20] W. Healy, C. Della, R. Iorio, K. Berend, F. Cushner, D. Dalury, J. Lonner, Complications of Total Knee Arthroplasty: standardized list and definitions of the Knee Society, Clin. Orthop. Relat. Res. 471 (2013) 215-220.

[21] C.R. Arciola, L. Visai, F. Testoni, S. Arciola, D. Campoccia, P. Speziale, L. Montanaro, Concise survey of Staphylococcus aureus virulence factors that promote adhesion and damage to peri-implant tissues, Int. J. Artif. Organs. 34 (2011) 771-780.

[22] N. Mas, I. Galiana, S. Hurtado, L. Mondragón, A. Bernardos, F. Sancenón, M.D. Marcos, P. Amorós, N. Abril-Utrillas, R. Martínez-Máñez, J.R. Murguía, Enhanced antifungal efficacy of tebuconazole using gated $\mathrm{pH}$-driven mesoporous nanoparticles, Int. J. Nanomedicine. 9 (2014) 2597-2606.

[23] L. Polo, N. Gómez-Cerezo, E. Aznar, J.-L. Vivancos, F. Sancenón, D. Arcos, M. Vallet-Regí, R. Martínez-Máñez, Molecular gates in mesoporous bioactive glasses for the treatment of bone tumors and infection, Acta Biomater. 50 (2017) 114-126. 
[24] S. Bagherifard, Mediating bone regeneration by means of drug eluting implants: From passive to smart strategies, Mater. Sci. Eng. C. 71 (2017) 1241-1252.

[25] N. Mas, D. Arcos, L. Polo, E. Aznar, S. Sánchez-Salcedo, F. Sancenón, A. García, M.D. Marcos, A. Baeza, M. Vallet-Regí, R. Martínez-Máñez, Towards the Development of Smart 3D "Gated Scaffolds" for On-Command Delivery, Small. 10 (2014) 4859-4864.

[26] D. Giacomini, P. Torricelli, G.A. Gentilomi, E. Boanini, M. Gazzano, F. Bonvicini, E. Benetti, R. Soldati, G. Martelli, K. Rubini, A. Bigi, Monocyclic $\beta$-lactams loaded on hydroxyapatite: New biomaterials with enhanced antibacterial activity against resistant strains, Sci. Rep. 7 (2017).

[27] L. Forte, P. Torricelli, F. Bonvicini, E. Boanini, G.A. Gentilomi, G. Lusvardi, E. Della Bella, M. Fini, E. Vecchio Nepita, A. Bigi, Biomimetic fabrication of antibacterial calcium phosphates mediated by polydopamine, J. Inorg. Biochem. 178 (2018) 43-53.

[28] E. Boanini, P. Torricelli, F. Bonvicini, M.C. Cassani, M. Fini, G.A. Gentilomi, A. Bigi, A new multifunctionalized material against multi-drug resistant bacteria and abnormal osteoclast activity, Eur. J. Pharm. Biopharm. 127 (2018) 120-129.

[29] I.A. Freires, C. Denny, B. Benso, S.M. De Alencar, P.L. Rosalen, Antibacterial activity of essential oils and their isolated constituents against cariogenic bacteria: A systematic review, Molecules. 20 (2015) 7329-7358.

[30] I.H.N. Bassolé, H.R. Juliani, Essential oils in combination and their antimicrobial properties, Molecules. 17 (2012) 3989-4006.

[31] S. Burt, Essential oils: their antibacterial properties and potential applications in foods - a review, Int. J. Food Microbiol. 94 (2004) 223-253.

[32] Z.E. Suntres, J. Coccimiglio, M. Alipour, The Bioactivity and Toxicological Actions of Carvacrol, Crit. Rev. Food Sci. Nutr. 55 (2015) 304-318.

[33] A. Rao, Y. Zhang, S. Muend, R. Rao, Mechanism of antifungal activity of terpenoid phenols resembles calcium stress and inhibition of the TOR pathway, Antimicrob. Agents Chemother. 54 (2010) 5062-5069.

[34] A. Ahmad, A. Khan, F. Akhtar, S. Yousuf, I. Xess, L.A. Khan, N. Manzoor, Fungicidal activity of thymol and carvacrol by disrupting ergosterol biosynthesis and membrane integrity against Candida, Eur. J. Clin. Microbiol. Infect. Dis. 30 (2011) 4150 .

[35] D.J. Fitzgerald, M. Stratford, M.J. Gasson, J. Ueckert, A. Bos, A. Narbad, Mode of antimicrobial of vanillin against Escherichia coli, Lactobacillus plantarum and Listeria innocua, J. Appl. Microbiol. 97 (2004) 104-113. 
[36] M. Ruiz-Rico, É. Pérez-Esteve, A. Bernardos, F. Sancenón, R. Martínez-Máñez, M.D. Marcos, J.M. Barat, Enhanced antimicrobial activity of essential oil components immobilized on silica particles, Food Chem. 233 (2017) 228-236.

[37] S. Ribes, M. Ruiz-Rico, É. Pérez-Esteve, A. Fuentes, P. Talens, R. MartínezMáñez, J.M. Barat, Eugenol and thymol immobilised on mesoporous silica-based material as an innovative antifungal system: Application in strawberry jam, Food Control. 81 (2017) 181-188.

[38] E.H. Cordes, W.P. Jencks, On the Mechanism of Schiff Base Formation and Hydrolysis, J. Am. Chem. Soc. 84 (1962) 832-837.

[39] J. Billman, A. Diesing, Reduction of Schiff bases with sodium borohydride, J. Org. Chem. 22 (1957) 1068-1070.

[40] K.J. Livak, T.D. Schmittgen, Analysis of relative gene expression data using real-time quantitative PCR and, Methods. 25 (2001) 402-408.

[41] V. Guarino, F. Causa, P.A. Netti, G. Ciapetti, S. Pagani, D. Martini, N. Baldini, L. Ambrosio, The role of hydroxyapatite as solid signal on performance of PCL porous scaffolds for bone tissue regeneration, J. Biomed. Mater. Res. - Part B Appl. Biomater. 86 (2008) 548-557. doi:10.1002/jbm.b.31055.

[42] A. Agostini, L. Mondragõn, A. Bernardos, R. Martínez-Máñez, M. Dolores Marcos, F. Sancenõn, J. Soto, A. Costero, C. Manguan-García, R. Perona, M. MorenoTorres, R. Aparicio-Sanchis, J.R. Murguía, Targeted cargo delivery in senescent cells using capped mesoporous silica nanoparticles, Angew. Chemie - Int. Ed. 51 (2012) 10556-10560.

[43] C. De La Torre, I. Casanova, G. Acosta, C. Coll, M.J. Moreno, F. Albericio, E. Aznar, R. Mangues, M. Royo, F. Sancenón, R. Martínez-Máñez, Gated mesoporous silica nanoparticles using a double-role circular peptide for the controlled and targetpreferential release of doxorubicin in CXCR4-expresing lymphoma cells, Adv. Funct. Mater. 25 (2015) 687-695.

[44] À. Ribes, E. Xifré-Pérez, E. Aznar, F. Sancenón, T. Pardo, L.F. Marsal, R. Martínez-Máñez, Molecular gated nanoporous anodic alumina for the detection of cocaine, Sci. Rep. 6 (2016).

\section{Figure captions}

Scheme 1. Synthetic protocol used to prepare vanillin derivative 3.

Figure 1. FESEM images of Sc, Sc-2 and Sc-3 surfaces. 
Figure 2. Cell viability after treating bacteria with different concentrations of functionalized microspheres (Pw-3).

Figure 3. Cell viability after treating $E$. coli bacteria with the functionalized scaffold (Sc-3), raw scaffold (Sc), vanillin (1) and modified vanillin (3) compared to a control (CTR).

Figure 4. FESEM images of bacteria treated with the non-functionalized scaffold (A) and with the functionalized scaffold (B, C, D).

Figure 5. Alamar blue assay (a), and LDH release (b) evaluated on L929 cells after 24 and 48 hours of culture in presence of $\mathbf{S c}, \mathbf{S c - 2}, \mathbf{S c - 3}$ and CTRs. Values are reported as mean $\pm \mathrm{SD}(* * *: \mathrm{p}<0.0005)$.

a) $* * *$ CTR+ vs Sc, Sc-2, Sc-3 and CTR- (24 and 48 hours);

b) $* * *$ CTR+ vs Sc, Sc-2, Sc-3 and CTR- (24 and 48 hours).

Figure 6. Neutral Red staining of L929 cells cultured in the presence of Sc, Sc-2, Sc-3, and CTR- and CTR+ after 24 and 48 hours (Magn x4).

Figure 7. Cell viability of MG63 on Sc, Sc-2, Sc-3 after 24 hours, 1 week, 2 weeks and 3 weeks of culture, quantified by Alamar blue and expressed as relative fluorescence units (RFU) $(* \mathrm{p}<0.05 ; * * \mathrm{p}<0.005 ; * * * \mathrm{p}<0.0005)$.

Sc: $* * * 24$ hours vs 1, 2, 3 weeks; ***1week vs 2, 3 weeks; Sc-3: ***24 hours vs 1, 2, 3 weeks; **1week vs 2, 3 weeks; Sc-2: **24 hours vs 1 week; ***24 hours vs 2, 3 weeks; *1week vs 2, 3 weeks.

Figure 8. LIVE/DEAD assay on MG63 seeded on Sc, Sc-2, Sc-3 and in control well after 24 hours, 1 week, 2 weeks and 3 weeks. Green fluorescence demonstrates both viability and distribution of the cells on the scaffolds. (Magn. 4x). 
Figure 9. Expression of typical osteoblast related genes during the in vitro culture:

ALPL (a), COL1A1 (b), BGLAP (c). No significant differences were evidenced.

Table 1. Summary of the solids used in this work.

\begin{tabular}{ccc}
\hline Name & Inorganic support & Organic attachment \\
\hline Pw & CaP powder & - \\
$\mathbf{P w}-\mathbf{3}$ & CaP powder & $\mathbf{3}$ \\
$\mathbf{S c}$ & CaP scaffold & - \\
Sc-2 & CaP scaffold & $\mathbf{2}$ \\
Sc-3 & CaP scaffold & $\mathbf{3}$ \\
\hline
\end{tabular}

Table 2. Amount of organic matter before and after the functionalization of different solids.

\begin{tabular}{cccccc}
\hline Material & Pw & Pw-3 & Sc & Sc-2 & Sc-3 \\
\hline $\mathrm{mg} \cdot \mathrm{g} \mathrm{solid}^{-1}$ & 0.078 & 6.861 & 1.055 & 3.136 & 3.463 \\
\hline
\end{tabular}

Table 3. $\mathrm{EC}_{50}$ of raw powder $\mathrm{CaP}$ material (Pw), functionalized $\mathrm{CaP}$ material (Pw-3), free vanillin, free diamine and free $\mathbf{3}$.

\begin{tabular}{cccccc}
\hline Material & Pw & Pw-3 & $\mathbf{1}$ & $\mathbf{2}$ & $\mathbf{3}$ \\
\hline $\mathrm{EC}_{50}\left(\mathrm{mg} \cdot \mathrm{mL}^{-1}\right)$ & - & 0.015 & 6.330 & 0.092 & 0.083 \\
\hline
\end{tabular}

